\title{
THE HOPF PROPERTY AND FREE PRODUCTS OF SEMIGROUPS
}

\author{
P. R. JONES \\ (Received 25 October 1978) \\ Communicated by T. E. Hall
}

\begin{abstract}
The free product of two Hopfian groups (in the category of groups) need not be Hopfian. We prove, by elementary methods, that the free product of two simple Hopfian inverse semigroups is Hopfian. In particular the free product of any two Hopfian groups, in the category of inverse semigroups, is again Hopfian. In fact the same is true in the category of all semigroups.
\end{abstract}

Subject classification (Amer. Math. Soc. (MOS) 1970): primary 20 M 05, secondary 08 A 10.

\section{Introduction}

An algebra is said to be Hopfian if each of its onto endomorphisms is an automorphism. Whether the Hopf property is preserved by free products has been considered for several classes of algebras. For example, Dey and Neumann (1970) showed that the free product of two finitely generated Hopfian groups is Hopfian. However, Newman and Sichler (1973) gave an example of two Hopfian groups whose free product is non-Hopfian. Each proof uses deep results on the structure of free products of groups.

In this paper we show, using only universality, that the free product of two simple Hopfian [inverse] semigroups, in the category of [inverse] semigroups, is again Hopfian. In particular this applies to any two Hopfian groups. (The general problem for inverse semigroups was posed by Reilly (1976), Question 15, in a widely circulated manuscript. It was incorrectly stated there that the general result for groups (in the category of groups) was in fact true.)

By way of examples we also show, in Section 3, that a simple inverse $\omega$-semigroup with Hopfian maximal subgroups is itself Hopfian. For simple inverse semigroups in general, however, this need not be so. 
For all relevant definitions and properties of semigroups the reader is referred to Clifford and Preston (1961, 1967). For an excellent bibliography on the Hopf property, see Hirshon (1975).

\section{Free products}

Let $\mathbf{K}$ be a class of semigroups (that is, $K$ is closed under isomorphism). If $U, V \in \mathbf{K}$ the free product of $U$ and $V$ in $K$ is the semigroup $S$ in $\mathbf{K}$, unique up to isomorphism if it exists, satisfying the usual universal properties in $\mathbf{K}$ : there exist monomorphisms $\eta_{U}: U \rightarrow S$ and $\eta_{V}: V \rightarrow S$ such that given any semigroup $T$ in $K$ and morphisms $\theta_{V}: U \rightarrow T$ and $\theta_{V}: V \rightarrow T$, there is a unique morphism $\theta: S \rightarrow T$ such that $\eta_{U} \theta=\theta_{U}$ and $\eta_{V} \theta=\theta_{V}$. When $S$ exists we may in the usual way identify $U$ and $V$ with their images in $S$. In that case $U$ and $V$ generate $S$ as a semigroup.

Proposition 1. Let $\mathbf{K}$ be a class of semigroups, $U, V \in \mathbf{K}$ and suppose they have free product $S$ in $\mathbf{K}$. If $\psi$ is an onto endomorphism of $S$ satisfying $U \psi=U$ and $V \psi=V$, then $\psi$ is an automorphism of $S$.

Proof. Since $U$ is Hopfian, $\psi \mid U$ is an automorphism of $U$ and therefore has an inverse $\theta_{U}$, again an automorphism of $U$. Similarly $\psi \mid V$ has an inverse $\theta_{V}$. By universality $\theta_{U}$ and $\theta_{V}$ extend to an onto endomorphism $\theta$ of $S$. Moreover $\psi \theta$ restricts to the identity on $U$ and $V$. By the uniqueness of such extension, $\psi \theta$ is the identity on $S$. Hence $\psi$ is injective, that is an automorphism.

We now develop some elementary properties of free products of semigroups. If $U$ and $V$ are any semigroups their 0 -direct sum is the semigroup $T=U \cup V \cup\{0\}$, the multiplication extending that on $U$ and $V$, all other products being zero.

LEMMA 2. Let $\mathbf{K}$ be a class of semigroups which is closed under 0-direct sums. Let $U, V \in \mathbf{K}$ and suppose they have free product $S$ in $\mathbf{K}$. Then

(i) $U$ is a union of $\mathscr{J}$-classes of $S$;

(ii) if $s \in S$ and $J_{s} \geqslant J_{u}$ for some $u$ in $U$, then $s \in U$.

Proof. Let $T$ be the 0 -direct sum of $U$ and $V$. Since $T \in \mathbf{K}$ and $T$ is generated by $U$ and $V$ there is a morphism $\alpha$ of $S$ upon $T$ extending the identity morphisms on $U$ and $V$. Suppose $J_{s} \geqslant J_{u}$ for some $s$ in $S, u$ in $U$. Then $J_{s \alpha} \geqslant J_{u \alpha}$ in $T$, where $u \alpha \in U$. From the definition of the multiplication in $T$, it is clear that $s \alpha \in U$. Hence $s \notin V$. If $s \notin U$ then $s$ can be expressed as a product involving elements of both $U$ and $V$, so that $s \alpha=0$. Therefore $s \in U$.

This proves (ii) and (i) is then immediate. 
Similar statements apply, of course, to $V$.

A semigroup is called simple if it contains no proper ideals or, equivalently, if it consists of a single $\mathscr{J}$-class.

LeMma 3. Let $\mathbf{K}$ be a class of semigroups closed under 0 -direct sums. Let $U, V \in K$ and suppose they have free product $S$ in $\mathbf{K}$. If $U$ and $V$ are simple and $\phi$ is an onto endomorphism of $S$ then $U \phi^{2}=U$ and $V \phi^{2}=V$.

Proof. Since $U$ and $V$ are simple each is a $\mathscr{J}$-class of $S$, by Lemma 2(i). Since $\phi$ is $\mathscr{J}$-preserving, $U \phi$ and $V \phi$ are therefore contained in $\mathscr{J}$-classes of $S$. Thus if, for example, $U \phi \cap U$ is non-empty, then $U \phi \subseteq U$.

Let $u \in U$ and $v \in V$. Then $x \phi=u$ and $y \phi=v$ for some $x, y$ in $S$. For $x$ there are three possibilities: either (i) $x \in U$, whence $U \phi \subseteq U$ (as above) or (ii) $x \in V$ whence $V \phi \subseteq U$, similarly, or (iii) $x$ is in neither $U$ nor $V$. In that case $x$ can be expressed as a product involving an element $s$ of $U$ and an element $t$ of $V$, so that $J_{s}>J_{x}$ and $J_{t}>J_{x}$. Then $J_{s \phi} \geqslant J_{u}$ and $J_{t \phi} \geqslant J_{u}$; so by Lemma 2(ii) $s \phi \in U$ and $t \phi \in U$. Hence in (iii) $U \phi \subseteq U$ and $V \phi \subseteq U$.

Similarly either $y \in U$, whence $U \phi \subseteq V$, or $y \in V$, whence $V \phi \subseteq V$, or finally $y$ is in neither $U$ nor $V$, whence $U \phi \subseteq V$ and $V \phi \subseteq V$. In any case either $U \phi \subseteq V$ or $V \phi \subseteq V$; so the case (iii) for $x$ is impossible. Hence either (i) $U \phi \subseteq U$, whence $V \phi \subseteq V$, or (ii) $V \phi \subseteq U$, whence $U \phi \subseteq V$. In either case $U \phi^{2} \subseteq U$ and $V \phi^{2} \subseteq V$.

Finally, noting that $\phi^{2}$ is again an onto endomorphism of $S$, let $u \in U$ and suppose $z \phi^{2}=u$, for some $z \in S$. Applying the above argument to $\phi^{2}$ yields $z \in U \cup V$. But $V \phi^{2} \subseteq V$, so $z \in U$. Hence $U \phi^{2}=U$ and similarly $V \phi^{2}=V$.

Now if $\phi^{2}$ is an automorphism of $S$ then so is $\phi$. So combining the lemma with Proposition 1 yields

THEOREM 4. Let $\mathbf{K}$ be a class of semigroups closed under 0-direct sums. If $U$ and $V$ are simple Hopfian semigroups in $\mathbf{K}$ then their free product in $\mathbf{K}$, if it exists, is Hopfian.

The three classes of immediate interest are the classes Sgp of all semigroups, Inv of inverse semigroups and $\mathbf{G p}$ of groups. (Note that every semigroup morphism between inverse semigroups is an inverse semigroup morphism, and similarly for groups, so that the Hopfian property is the same in each class.) It is well known (see, for example, Preston (1973), McAlister (1976)) that each class has the free product, denoted here by $U \operatorname{sgp} V, U$ inv $V$ and $U$ gp $V$ respectively, of any semigroups $U$ and $V$ in the respective classes. Further the 0-direct sum of two inverse semigroups is again inverse (though of course the same is not true for groups!). Thus we have our main theorem. 
TheOREM 5. If $U$ and $V$ are simple Hopfian semigroups then $U \operatorname{sgp} V$ is Hopfian. If in addition $U$ and $V$ are inverse then $U$ inv $V$ is Hopfian.

Since any group is a simple inverse semigroup we have the corollaries stated in the Introduction.

Corollary 6. If $G$ and $H$ are Hopfian groups then $G \operatorname{sgp} H$ and $G \operatorname{inv} H$ are Hopfian.

\section{Examples}

Apart from the Hopfian groups, simple Hopfian (inverse) semigroups are plentiful: any simple inverse $\omega$-semigroup whose maximal subgroups are Hopfian is itself Hopfian (Theorem 7 below). For arbitrary simple inverse semigroups, however, this is no longer so (Example 8).

Recall that an inverse semigroup $S$ is an w-semigroup if its semilattice of idempotents is isomorphic to $C_{\omega}$, the (chain of) positive integers under the inverse of their usual order. Munn (1968) and Kočin (1968) independently showed how to construct all simple inverse $\omega$-semigroups from groups and morphisms between them. Using our result, examples of Hopfian simple inverse $\omega$-semigroups with any finite number of $\mathscr{D}$-classes can easily be constructed. To prove the result, though, we will not need the explicit structure of a simple inverse $\omega$-semigroup, only the fact that it has a finite number $d$, say, of $\mathscr{D}$-classes, each of which is a bisimple inverse $\omega$-semigroup; the idempotents can then be written as follows:

$$
\begin{aligned}
& e_{1}>e_{2}>\ldots>e_{d} \\
> & e_{d+1}>e_{d+2}>\ldots>e_{2 d} \\
> & e_{2 d+1}>\ldots \\
> & \ldots
\end{aligned}
$$

where the idempotents in any column form the set of idempotents of an entire $\mathscr{D}$-class.

THEOREM 7. A simple inverse w-semigroup is Hopfian if each of its maximal subgroups is.

Proof. Let $T$ be a simple inverse $\omega$-semigroup with $d \mathscr{D}$-classes, $d \in \mathbf{N}$, and semilattice of idempotents $E$. Let $\phi$ be an onto endomorphism of $T$ and put $\alpha=\phi \phi^{-1}$, a congruence on $T$ satisfying $T / \alpha \cong T$. Denote by $\alpha^{E}$ the restriction of $\alpha$ to the idempotents of $T$. 
If $d=1$, then $T$ is bisimple and by Munn and Reilly (1966) $\alpha$ is either a group congruence or is idempotent-separating (that is, $\alpha^{E}$ is trivial). Clearly the latter case holds.

If $d \geqslant 2$, suppose $\alpha^{E}$ is non-trivial. Then $\left(e_{i}, e_{j}\right) \in \alpha$ for some $i<j$ and so $\left(e_{j-1} e_{i}, e_{j-1} e_{j}\right) \in \alpha$, that is $\left(e_{j-1}, e_{j}\right) \in \alpha$. But $e_{j-1}$ and $e_{j}$ lie in different $\mathscr{D}$-classes of $T$ and so $T / \alpha$ has at most $d-1 \mathscr{D}$-classes, contradicting $T / \alpha \cong T$. Hence $\alpha$ is again idempotent-separating.

So $\phi$ is also idempotent-separating and $\phi \mid E$ is an automorphism. However it is easy to show that $E$ is rigid, that is $\operatorname{Aut}(E)$ is trivial. Hence $\phi \mid E$ is the identity on $E$ and so for each $e$ in $E, H_{e} \phi \subseteq H_{e}$. In fact $H_{e} \phi=H_{e}$. (If $s \in T$ and $s \phi \in H_{e}$, then $\left(s s^{-1}\right) \phi=\left(s^{-1} s\right) \phi=e=e \phi$. So $s s^{-1}=s^{-1} s=e$, that is $s$ is in $H_{e}$.) Since $H_{e}$ is a maximal subgroup and therefore Hopfian, $\phi \mid H_{e}$ is an automorphism of $H_{e}$. Thus $e \alpha=\{e\}$ for each $e$ in $E$. But a congruence on an inverse semigroup is determined by its classes containing idempotents. Hence $\alpha$ is trivial, that is $\phi$ is an automorphism of $T$.

We conclude by showing that the statement of Theorem 7 is not true for simple, or even bisimple, inverse semigroups in general. For completeness we will include some preliminaries.

Bruck (1958) showed that any semigroup $U$ can be embedded in a simple semigroup, which we denote $\mathscr{C}(U)$, having the following properties (Clifford and Preston (1967), Section 8.5; see also Howie (1976), Section V.6):

(i) $\mathscr{C}(U)$ is inverse if and only if $U$ is,

(ii) if $U$ is trivial then $\mathscr{C}(U)$ is the bicyclic semigroup,

(iii) the $\mathscr{L}$-, $\mathscr{R}$ - and $\mathscr{D}$-classes of $\mathscr{C}(U)$ are in one-to-one correspondence with those of $U$, so that

(iv) $\mathscr{C}(U)$ is bisimple if and only if $U$ is, and

(v) $\mathscr{C}(U)$ is combinatorial (that is $\mathscr{H}$ is trivial) if and only if $U$ is,

(vi) every morphism of $U$ upon $V$ induces a morphism of $\mathscr{C}(U)$ upon $\mathscr{C}(V)$ such that the following diagram commutes:

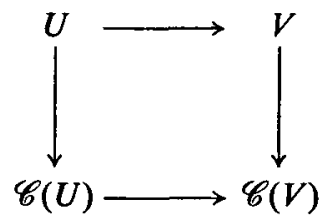

It is immediate from (vi) that if $U$ is non-Hopfian, so is $\mathscr{C}(U)$. Thus if for $U$ we choose, for example, the semilattice $C_{\omega}$, then $\mathscr{C}\left(C_{\omega}\right)$ is a non-Hopfian simple inverse semigroup with trivial subgroups, by (v). (Note that $\mathscr{C}\left(C_{\omega}\right)$ has infinitely many $\mathscr{D}$-classes.) 
Now suppose $U_{1}$ is an arbitrary bisimple inverse semigroup, put $U_{2}=\mathscr{C}\left(U_{1}\right)$, $U_{3}=\mathscr{C}\left(U_{2}\right)$ and inductively $U_{i+1}=\mathscr{C}\left(U_{i}\right)$, with associated embedding $\theta_{i}: U_{i} \rightarrow U_{i+1}$, for all $i \geqslant 1$. Clearly each $U_{i}$ is a bisimple inverse semigroup. Let $T$ be the direct limit (see for example McAlister (1968)) of the system:

$$
U_{1} \stackrel{\theta_{1}}{\longrightarrow} U_{2} \stackrel{\theta_{2}}{\longrightarrow} \ldots \longrightarrow U_{i} \stackrel{\theta_{i}}{\longrightarrow} U_{i-1} \longrightarrow \ldots .
$$

It is routine to verify that $T$ is again a bisimple inverse semigroup. Further $T$ is combinatorial if and only if $U_{1}$ is.

Suppose there is also a morphism $\phi_{1}$ of $U_{2}$ upon $U_{1}$. Then from (vi) above $\phi_{1}$ induces a morphism $\phi_{2}$, say, of $\mathscr{C}\left(U_{2}\right)$ upon $\mathscr{C}\left(U_{1}\right)$, that is of $U_{3}$ upon $U_{2}$. Inductively, there is a morphism $\phi_{i}$ of $U_{i+1}$ upon $U_{i}$ for every $i \geqslant 1$, so that the following diagram commutes:

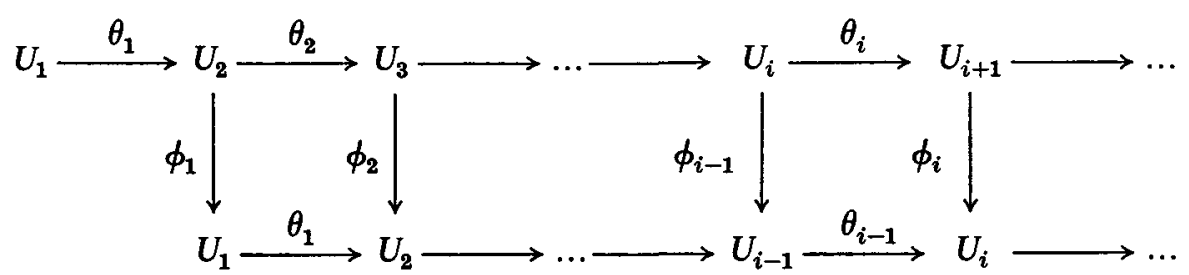

From the universal properties of the direct limit it is apparent that there is an onto endomorphism $\phi$ of $T$ 'extending' the $\phi_{i}$ 's, for all $i \geqslant 1$.

Finally take $U_{1}=B$, the bicyclic semigroup, in this construction, with $\phi_{1}$ the morphism of $U_{2}=\mathscr{C}(B)$ upon $B$ induced by the trivial morphism $B \rightarrow\{e\}$, where $e$ is the identity of $B$. (By (ii) above, $\mathscr{C}(e) \cong B$ ). Clearly $\phi_{1}$ is not one-to-one. Hence the construction yields a semigroup of the following type.

EXAMPLE 8. There exists a non-Hopfian bisimple inverse semigroup whose maximal subgroups are Hopfian, in fact trivial.

Note ADDed In Proof. In a future paper of a similar title the author will extend the methods of this paper to free products of various finitely generated semigroups.

\section{References}

R. H. Bruck (1958), A survey of binary systems (Ergebnisse der Mathematik, Heft. 20, Springer, Berlin).

A. H. Clifford and G. B. Preston (1961), Algebraic theory of semigroups, Vol. I, Math. Surveys 7 (American Math. Soc., Providence, R.I.).

A. H. Clifford and G. B. Preston (1967), Algebraic theory of semigroups, Vol. II, Math. Surveys 7 (American Math. Soc., Providence, R.I.). 
I. M. S. Dey and H. Neumann (1970), 'The Hopf property of free products', Math. Z. 117, 325-339.

R. Hirshon (1975), 'The intersection of the subgroups of finite index in some finitely presented groups', Proc. Amer. Math. Soc. 53, 32-36.

J. M. Howie (1976), An introduction to semigroup theory (Academic Press, London).

B. P. Kočin (1968), 'The structure of inverse ideal-simple $\omega$-semigroups', Vestnik Leningrad Univ. Math. 23, 41-50 (in Russian).

D. B. McAlister (1968), 'A homomorphism theorem for semigroups', J. London Math. Soc. 43, 355-366.

D. B. McAlister (1976), 'Inverse semigroups generated by a pair of subgroups', Proc. Roy. Soc. Edinburgh Sect. A 77A, 9-22.

W. D. Munn (1968), 'Regular w-semigroups', Glasgow Math. J. 9, 46-66.

W. D. Munn and N. R. Reilly (1966), 'Congruences on a bisimple $\omega$-semigroup', Proc. Glasgow Math. Assoc. 7, 184-192.

M. F. Newman and J. Sichler (1973), 'Free products of Hopf groups', Math. Z. 135, 69-72.

G. B. Preston (1973), 'Inverse semigroups: some open questions', Proc. Symposium on Inverse Semigroups and Their Generalizations, pp. 122-139 (Northern Illinois University).

N. R. Reilly (1976), 'Free inverse semigroups', manuscript.

Mathematics Department

University of Western Australia

Nedlands, Western Australia

Australia 6009 